\title{
Pneumonia caused by Talaromyces marneffei in an epidermal growth factor receptor (EGFR) mutation-positive advanced lung adenocarcinoma patient: a case report
}

\author{
Wei Liu ${ }^{1 \#}$, Jinhe $\mathrm{Xu}^{2 \#}$, Baoquan Lin ${ }^{3}$, Yabin Zhang ${ }^{4}$, Feilai Xie ${ }^{5}$, Chengzhi Zhou ${ }^{6}$, Guoxiang Lai ${ }^{1}$, \\ Lei Zhang', Zongyang Yu' \\ ${ }^{1}$ Department of Respiratory and Critical Care Medicine, Dongfang Hospital of Xiamen University, Fuzhou General Hospital of Fujian Medical \\ University, The 900th Hospital of the Joint Logistic Support Force, PLA, Fuzhou, China; ${ }^{2}$ Graduate College of Fujian Medical University, Fuzhou, \\ China; ${ }^{3}$ Department of Cardio-Thoracic Surgery, Dongfang Hospital of Xiamen University, Fuzhou General Hospital of Fujian Medical University, \\ The 900th Hospital of the Joint Logistic Support Force, PLA, Fuzhou, China; ${ }^{4}$ Institute for Laboratory Medicine, Dongfang Hospital of Xiamen \\ University, Fuzhou General Hospital of Fujian Medical University, The 900th Hospital of the Joint Logistic Support Force, PLA, Fuzhou, China; \\ ${ }^{5}$ Pathology Department, Dongfang Hospital of Xiamen University, Fuzhou General Hospital of Fujian Medical University, The 900th Hospital \\ of the Joint Logistic Support Force, PLA, Fuzhou, China; ${ }^{6}$ State Key Laboratory of Respiratory Disease, National Clinical Research Center for \\ Respiratory Disease, Guangzhou Institute of Respiratory Health, the First Affiliated Hospital of Guangzhou Medical University, Guangzhou, China \\ \#These authors contributed equally to this work. \\ Correspondence to: Zongyang Yu. Department of Respiratory and Critical Care Medicine, Dongfang Hospital of Xiamen University, Fuzhou General \\ Hospital of Fujian Medical University, The 900th Hospital of the Joint Logistic Support Force, PLA, Fuzhou, China. Email: yuzy527@sina.com.
}

\begin{abstract}
Talaromyces marneffei (T. marneffei) is a pathogenic, thermally dimorphic fungus that can cause invasive infection and significant morbidity in immunocompromised patients, especially those with human immunodeficiency virus (HIV) or other immune defects. Currently, T. marneffei infection is understood to be not limited only to immunodeficient patients, as cases of immunocompromised patients or immunocompetent patients associated with T. marneffei infection have been increasingly reported in recent years. The exact mechanism is not yet clear. This study reports a case of an advanced lung adenocarcinoma patient with T. marneffei infection. The patient is a 59-year-old female with a 3 -month history of coughing, expectoration, and progressive dyspnea. Computed tomography (CT) scans showed a mass in the left lower lung, multiple plaques and nodules in both lungs, and left pleural effusion. The patient was diagnosed with T. marneffei infection, as T. marneffei was found in both the bronchoalveolar lavage fluid (BALF) and the sputum. According to the pathology of the left lung lesion by transbronchial lung biopsy (TBLB) and contrast-enhanced brain magnetic resonance imaging (MRI), the patient was diagnosed with epidermal growth factor receptor (EGFR) mutation-positive stage IV lung adenocarcinoma (T4N3M1c). She received intravenous liposomal amphotericin B and oral itraconazole as anti-fungal treatments, meanwhile, icotinib was used as an anti-tumor treatment. Following treatment, CT re-examination showed that the mass was remarkably absorbed, and some of the lung nodules had disappeared. No relapse of T. marneffei infection was found during the follow-up. This case indicates that patients with malignant lung tumors may possibly become infected with T. marneffei. Sequential treatment of amphotericin liposome B followed by itraconazole is effective for lung cancer patients with T. marneffei infection.
\end{abstract}

Keywords: Case report; Talaromyces marneffei (T. marneffei); human immunodeficiency virus-negative (HIVnegative); lung adenocarcinoma

\footnotetext{
$\wedge$ ORCID: 0000-0001-6060-4306.
} 
Submitted Sep 24, 2020. Accepted for publication Dec 02, 2020.

doi: 10.21037/apm-20-2137

View this article at: http://dx.doi.org/10.21037/apm-20-2137

\section{Introduction}

Talaromyces marneffei (T. marneffei, previously known as Penicillium marneffei) is a major fungal pathogen endemic to Southeast Asia. It causes Talaromycosis (formerly known as Penicilliosis), an invasive infection that manifests as either local or disseminated infection, which is mainly dependent on the immune status of the host. As a thermally dimorphic pathogen, colonies of T. marneffei are yellowishgreen with diffusing red pigment when incubated at room temperature; however, when incubated at $37^{\circ} \mathrm{C}$, the colonies become white, dry, and yeast-like. Since the discovery of T. marneffei in 1950s, our knowledge of this fungus has been greatly improved, despite still lacking a comprehensive understanding. Certain bamboo rat species have been considered as natural carriers of T. marneffei (1). At present, a sufficient explanation regarding the route of transmission of the disease is unavailable, although infection possibly occurs via skin damage, the digestive tract, or inhalation of conidia.

$\mathrm{HIV}$-infected patients with a cluster of differentiation (CD4) cell count of $<100$ cells $/ \mu \mathrm{L}$ (2) are predominantly susceptible to Talaromycosis, however, cases of $\mathrm{T}$. marneffei infection in non-HIV-infected patients have been increasingly reported in recent years (3). Non-HIV-infected patients are defined as those who are immunodeficient, immunocompromised, or immunocompetent, including primary immunodeficiency (PID) pediatric patients (4), patients with adult-onset immunodeficiency syndrome caused by anti-interferon-gamma (anti-IFN- $\gamma$ ) autoantibodies (5), patients with solid organ and hematopoietic stem cell transplantation, patients with autoimmune diseases, malignant tumors (6-8), or chronic obstructive pulmonary disease (COPD) (9), and even previously healthy patients (10).

There has been no reported case of Talaromyces marneffei infection in targeted therapy patients. The present study reports a case of an advanced epidermal growth factor receptor (EGFR) mutation-positive lung adenocarcinoma patient with pneumonia caused by $\mathrm{T}$. marneffei.

We present the following article in accordance with the CARE reporting checklist (available at http://dx.doi. org/10.21037/apm-20-2137).

\section{Case presentation}

A 59-year-old female with a 3-month history of productive cough and progressive dyspnea attended our hospital for treatment on May 15, 2020. The patient suffered from obvious breathing difficulty, especially during physical labor or in fatigue, but presented without fever, chest pain, palpitations, or weight loss. She denied any recent travel or contact with bamboo rats. From May 3 to May 14, she was treated at a local hospital. Chest computed tomography (CT) scans showed a mass in the left lower lung, multiple plaques and nodules in both lungs, and left pleural effusion (Figure 1). She underwent closed thoracic drainage, and enclosure pneumothorax was developed postoperatively. She was transferred to our hospital for further diagnosis and treatment.

We performed a physical examination of the patient upon admission. Her body temperature was $36.2^{\circ} \mathrm{C}$. The closed thoracic drainage tube in the left front chest was unobstructed and without crackles in both lungs.

During admission, she underwent routine blood, sputum, pleural fluid, urine, and stool laboratory tests. Chest CT scan and bronchoscopy with transbronchial lung biopsy (TBLB) and bronchoalveolar lavage (BAL) were also performed. Her blood was HIV-negative, and she had normal CD4 $(905$ cells $/ \mu \mathrm{L})$ and cluster of differentiation 8 (CD 8$)(410$ cells $/ \mu \mathrm{L})$ counts. Furthermore, arterial blood gas was analyzed $\left(\mathrm{FiO}_{2}=0.21\right)$, and the results were as follows: $\mathrm{pH}$ of 7.44 , partial pressure of oxygen $\left(\mathrm{PO}_{2}\right)$ of $86 \mathrm{mmHg}$, partial pressure of carbon dioxide $\left(\mathrm{pCO}_{2}\right)$ of $41 \mathrm{mmHg}$, standard bicarbonate $\left(\mathrm{HCO}_{3}\right.$-std) of $27.5 \mathrm{mmol} / \mathrm{L}$, oxygen saturation $\left(\mathrm{SaO}_{2}\right)$ of $97 \%$, and oxygenation index of 409 . Moreover, the patient's white blood cell count was normal [total count: $6.14 \times 10^{9} / \mathrm{L}\left(3.5-9.5 \times 10^{9} / \mathrm{L}\right)$, neutrophil count: $3.41 \times 10^{9} / \mathrm{L}$ (1.8-6.3×10\%/L), neutrophils: $55.6 \%$ (40-75\%), lymphocytes count: $2.09 \times 10^{9} / \mathrm{L}\left(1.1-3.2 \times 10^{9} / \mathrm{L}\right)$, lymphocytes: $34 \%(20-$ $50 \%)$. Also, hemoglobin was $122 \mathrm{mg} / \mathrm{L}(115-150 \mathrm{mg} / \mathrm{L})$, platelets were $314 \times 10^{9} / \mathrm{L}\left(125-350 \times 10^{9} / \mathrm{L}\right)$, C-reactive protein $(\mathrm{CRP})$ was $<7.2 \mathrm{mg} / \mathrm{L}(0-8 \mathrm{mg} / \mathrm{L})$, erythrocyte sedimentation rate (ESR) was $5 \mathrm{~mm} / \mathrm{h}(<15 \mathrm{~mm} / \mathrm{h})$, and procalcitonin (PCT) was normal $(<0.05 \mathrm{ng} / \mathrm{mL})$. The $1,3-$ $\beta-\mathrm{D}$ glucan $(\mathrm{G})$ and galactomannan $(\mathrm{GM})$ tests of serum, $\mathrm{G}$ test of bronchoalveolar lavage fluid (BALF), and the 
2020-05-15
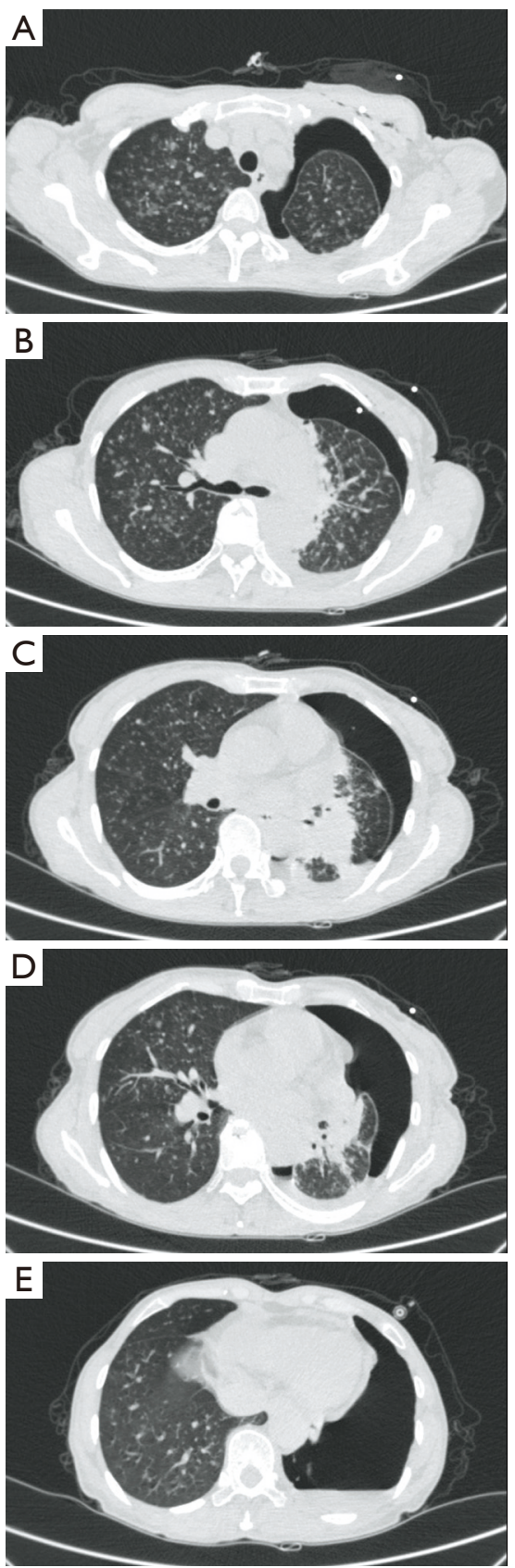

2020-05-27
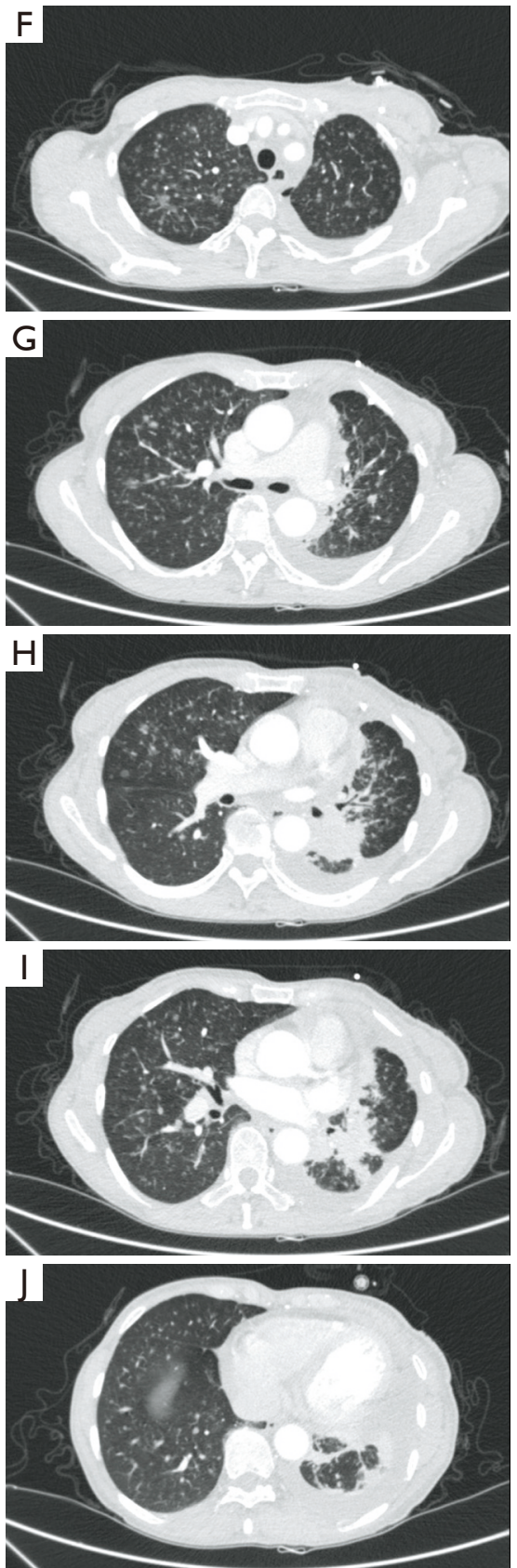

2020-08-18
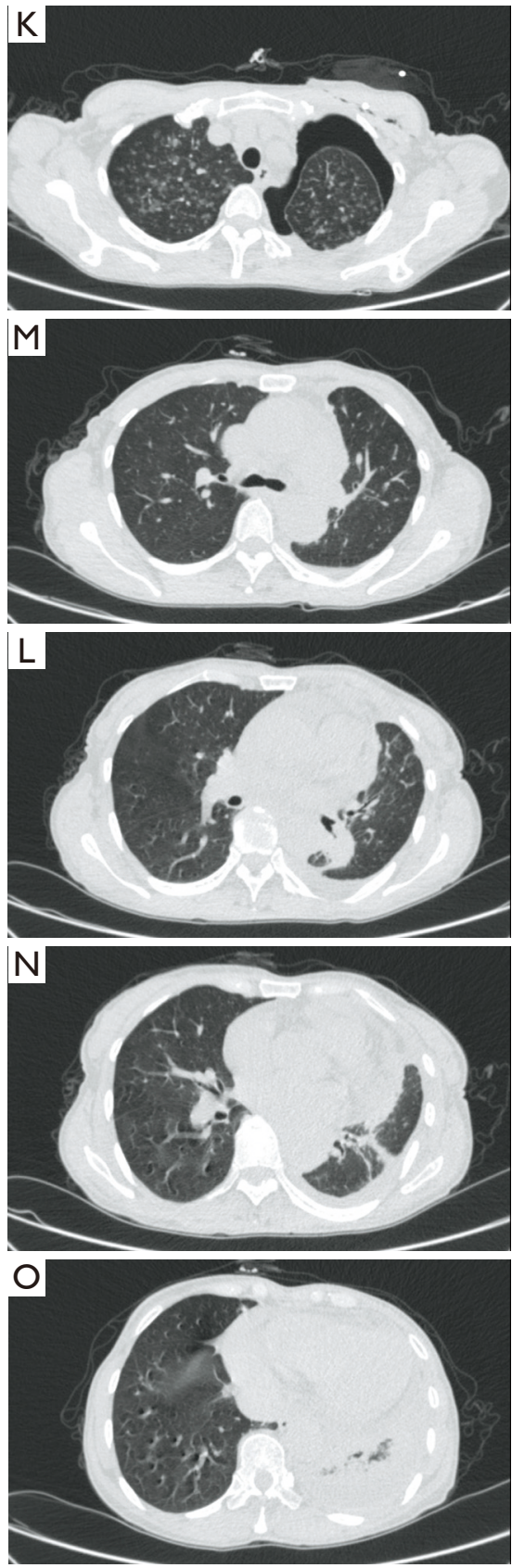

Figure 1 On admission (2020-05-15), Chest computed tomography (CT) scan showed the mass in the left lower lung accompanied by multiple nodes throughout both lungs and hydropneumothorax (A,B,C,D,E). On 2020-05-27, chest CT scan showed that most of the pneumothorax was absorbed (F,G,H,I,J). On 2020-08-18 (K,L,M,N,O), chest CT scan showed that most of the multiple nodes throughout both lungs had disappeared and the size of the mass in the left lower lung was also reduced following treatment. 

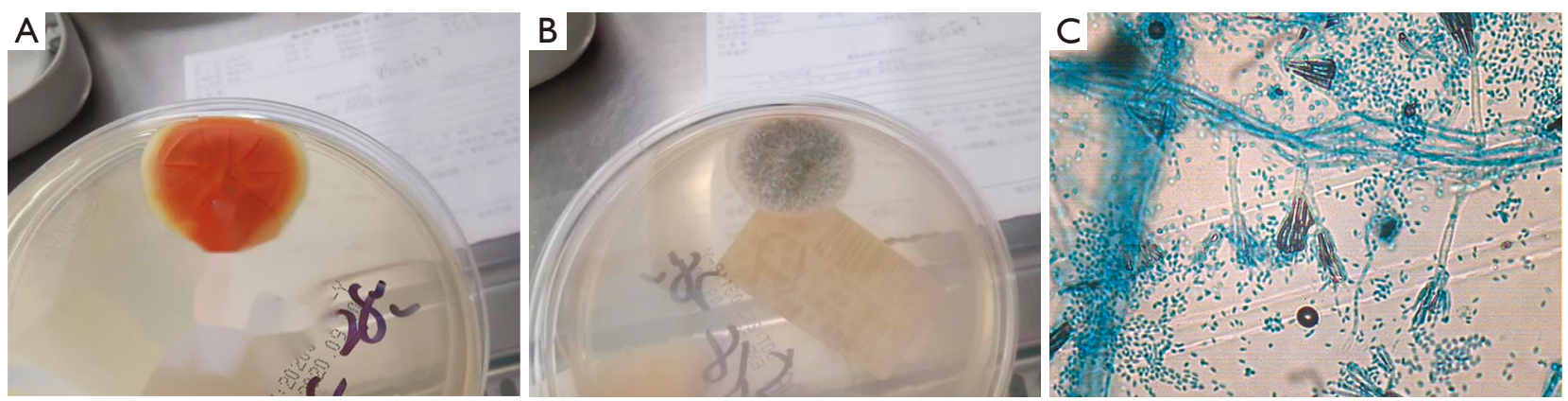

Figure 2 One yellowish-green colony with diffusing red pigment. (A,B) Sputum sample fungus culture in Sabouraud-glucose agar after incubation at room temperature. (C) Mycelium presented double-helical broom-like branches, scattered, slightly asymmetric under a light microscope (Staining with lactophenol cotton blue, $\times 40$ ).

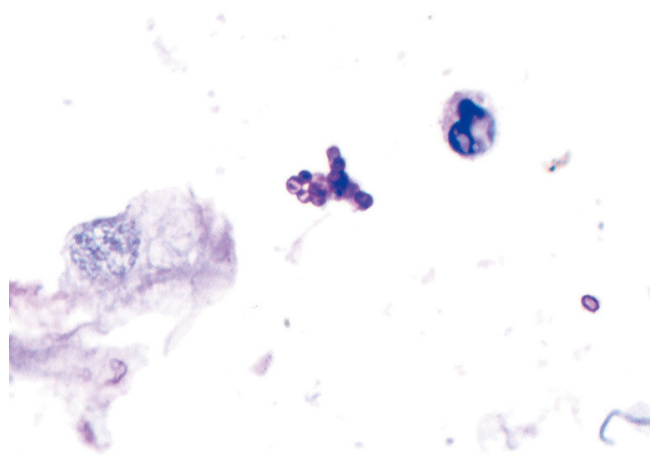

Figure 3 Several periodic acid-Schiff (PAS)-positive yeast-like organisms with a central septum were identified by bronchoalveolar lavage fluid (BALF) cytology examination (periodic acid Schiff reaction, PAS reaction, $\times 1,000)$.

acid-fast bacillus test of the sputum and BALF were all negative. Pleural fluid fungi and bacterial cultures were also negative. Moreover, blood spartate aminotransferase, blood alanine aminotransferase, blood creatinine, blood urea nitrogen, blood sugar, and serum electrolytes were all normal.

The GM test of BALF was positive. One yellowishgreen colony with diffusing red pigment was detected in the patient's sputum sample by fungi culture incubated at room temperature (Figure 2). BALF cytology examination identified some periodic acid-Schiff (PAS)- and periodic acid methenamine silver (PAM)-positive yeast-like organisms with a central septum (Figure 3). Histological examination (HE) of the left lung lesion by TBLB indicated that the patient had non-small cell lung cancer (NSCLC) with a possibility of transforming into adenocarcinoma (Figure 4).
Immunohistochemistry revealed that antigen $\mathrm{Ki}-67$ (15\%), thyroid transcription factor 1 (TTF-1) (+++), and Napsin A (+++) were positive, while programmed death-ligand 1 (PD-L1) was negative. Next generation sequencing (NGS) detection of the biopsy tissues by TBLB showed EGFR p.Glu746_Ala750del(E19). Heterosexual cells were detected in the pleural fluid.

Chest CT revealed a mass in the left lower lung, multiple nodes in both lungs, and hydropneumothorax (Figure $1 A, B, C, D, E)$. Brain metastasis was observed by contrast material-enhanced magnetic resonance imaging (MRI) of the brain (Figure 5). No abnormalities of the upper abdomen were found by contrast material-enhanced CT or whole body bone emission CT (ECT). Based on the above results and observations, the patient was diagnosed with a $T$. marneffei lung infection and EGFR mutation-positive stage IV lung adenocarcinoma (T4N3M1c).

Following admission, a pressure-attracting implement was connected to a closed thoracic drainage tube to prevent air leakage. After being diagnosed with a T. marneffei infection, the patient received intravenous liposomal amphotericin B as an anti-fungal treatment followed by oral icotinib as an anti-tumor treatment. The patient's conditions improved after 2 weeks of treatment, and she was prescribed oral itraconazole and icotinib therapy and discharged. The initial dose of liposomal amphotericin B was $5 \mathrm{mg} /$ day daily, increased by 5 to $40 \mathrm{mg} /$ day. However, when the dose was increased to $50 \mathrm{mg} /$ day, the patient developed hypokalemia, which is a common side effect of liposomal amphotericin B. Intravenous dexamethasone was used for pretreatment prior to each intravenous injection of liposomal amphotericin B.

Following treatment, CT re-examination showed that 


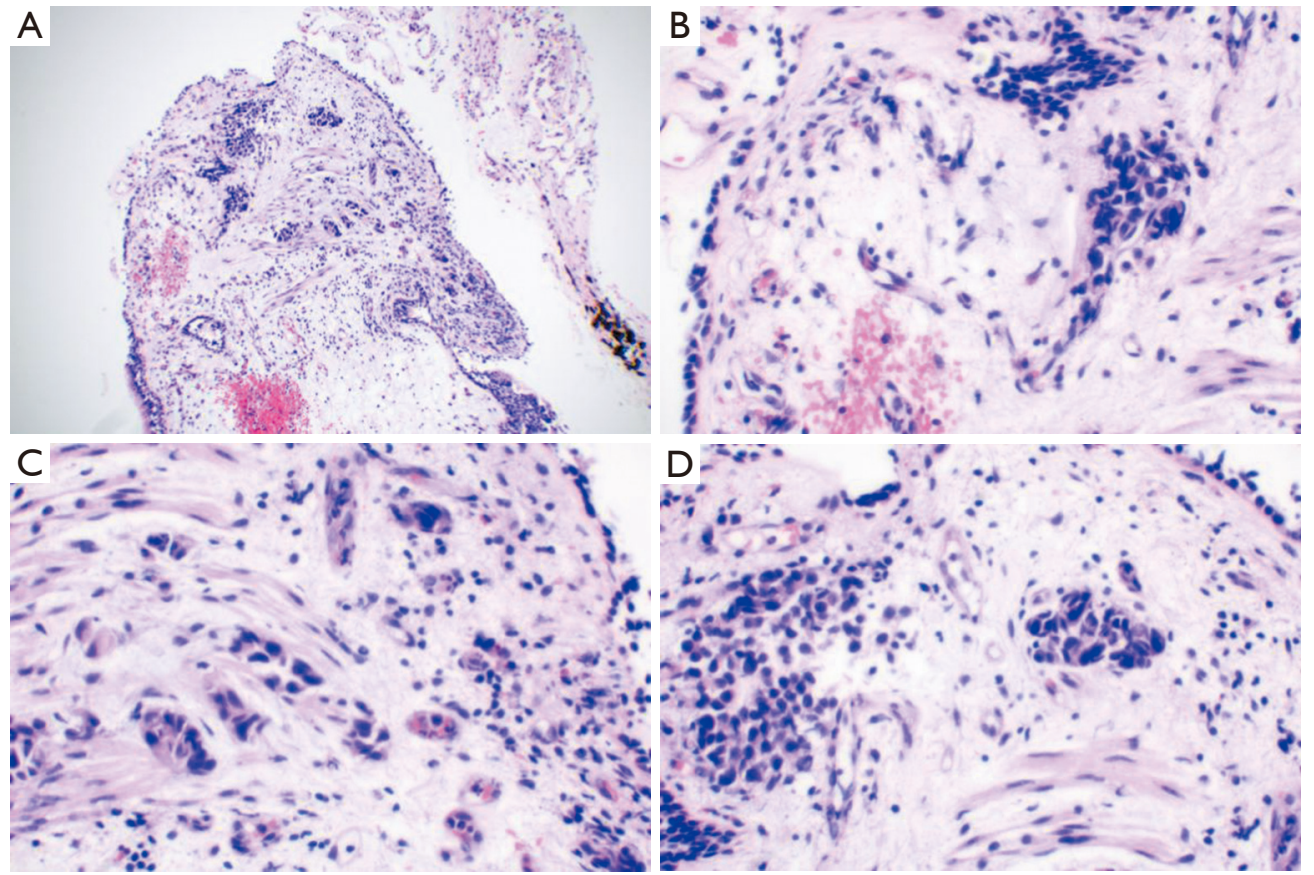

Figure 4 The Histological examination (HE) film of the left lung lesion by transbronchial lung biopsy (TBLB) showed infiltration of glandular tissues, indicating the possibility of non-small cell lung cancer transforming into adenocarcinoma (hematoxylin and eosin stain, A: $\times 100, \mathrm{~B} / \mathrm{C} / \mathrm{D}: \times 400)$.

the mass was significantly absorbed and most lung nodules disappeared, however, pleural effusion was unchanged (Figure 1). Until Nov 5, 2020, follow-up showed no relapse of $\mathrm{T}$. marneffei infection or tumor progression. All procedures performed in studies involving human participants were in accordance with the ethical standards of the institutional and/or national research committee(s) and with the Helsinki Declaration (as revised in 2013). Written informed consent was obtained from the patient.

\section{Discussion and conclusions}

The present study reports a local infection case of Talaromycosis in a non-HIV-infected patient with advanced lung adenocarcinoma. The patient had normal CD4 and CD8 counts, which suggested normal cell immunity and explained why the infection was not disseminated. Our literature review identified few cases of lung cancer with Talaromycosis $(8,11)$. Lung cancer will impair the immune system, but whether this is an important cause of Talaromycosis remains to be confirmed by future studies. Although the patient denied any recent travel or contact with bamboo rats, T. marneffei could possibly exist in some environmental reservoirs, such as soil (1).

Clinical manifestations of T. marneffei infection vary, and its severity depends on the immune status of patient, as well as the time of diagnosis and treatment. Disseminated infections are usually associated with immunodeficient patients, including patients with HIV/AIDS, PIDs, or impaired cellular immunity. The symptoms and signs of disseminated $T$. marneffei infection are both systemic and local. In addition to fever, weight loss, and fatigue, depending on the organ systems invaded by the fungus, the specific symptoms and signs include erythra or skin lesions, hepatosplenomegaly, lymphadenopathy, blurred vision, redness and foreign body sensation, and respiratory and gastrointestinal abnormalities. Cases of local infection cases typically exhibit localized symptoms and signs, and rarely involve systemic symptoms.

The patient in the current study presented with fatigue, coughing and sputum production, exertional dyspnea, and progressive dyspnea mainly due to growing pleural effusion, but did not have a fever, which is also common in infectious diseases. Coughing is not specific to a certain lung disease, as it could have been caused by both lung cancer and/or infectious lung disease in the current case. However, sputum 

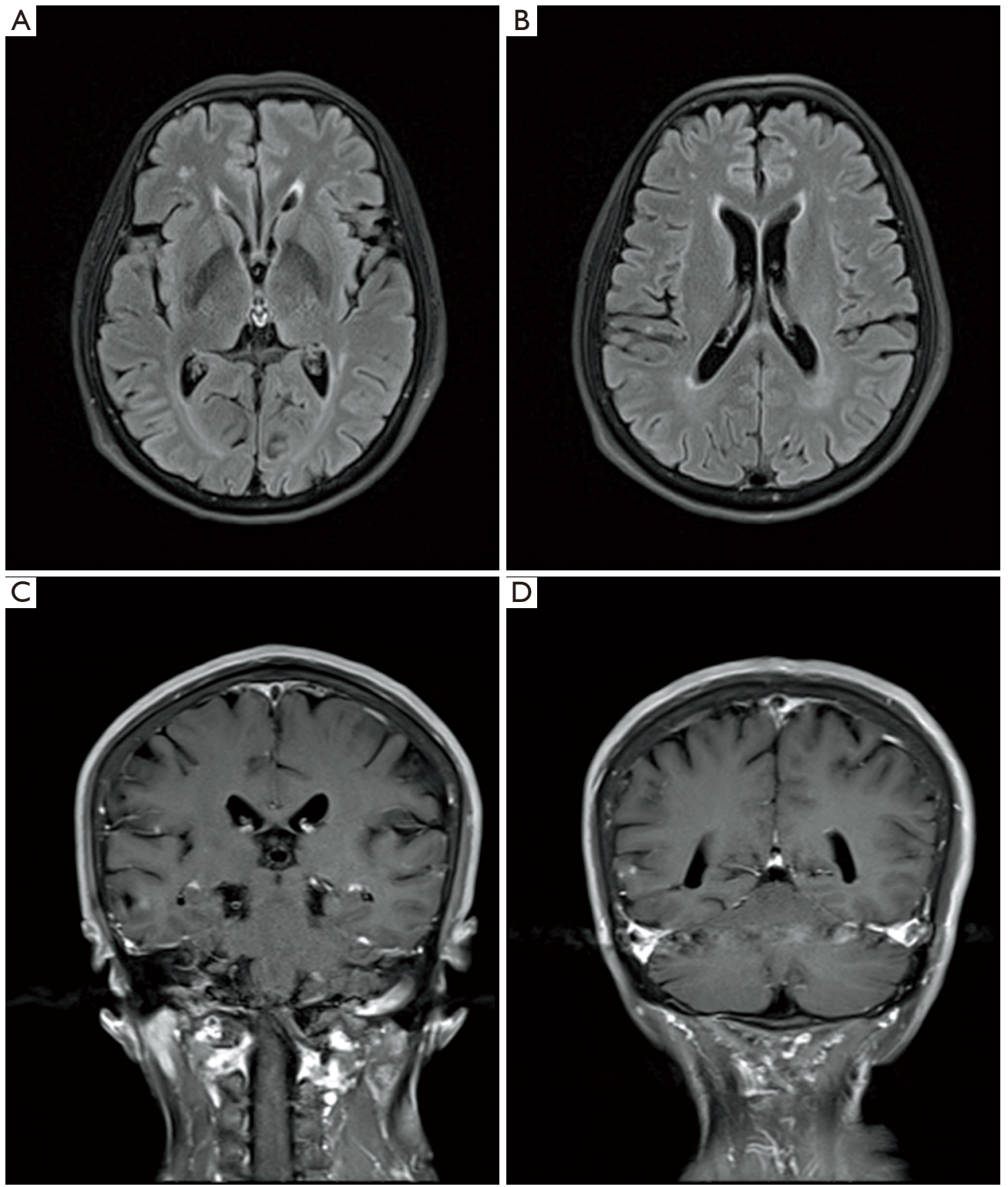

Figure 5 On admission (2020-05-26), brain metastasis was found by contrast material-enhanced brain magnetic resonance imaging (MRI) of the brain $(\mathrm{A}, \mathrm{B}, \mathrm{C}, \mathrm{D})$.

production is commonly observed in obstructive pneumonia or complicated infectious lung diseases caused by lung cancer.

Mycological culture of body fluids or tissues is a highly effective method for the diagnosis of T. marneffei infection. Bone marrow, skin biopsy, and blood are the three samples with the highest sensitivity in the disseminated infection (12). The current case is a local infection in the lung, and T. marneffei was identified based on the typical morphology of colonies in the sputum samples cultured and the microscopic morphology of the BALF cytology examination. Therefore, T. marneffei was diagnosed as the cause of the patient's pneumonia.

At the time of admission, there were multiple nodules in the patient's lungs, some of which were solid and others that were ground-glass on the chest CT film. However, whether these nodules were metastatic cancer nodules or infected lesions was difficult to confirm. According to the CT findings of the chest during the follow-up (Figure $1 F, G, H, I, \mathcal{F}, K, L, M, N, O)$, most of the nodules had 
disappeared within a short period of time following antiinfective treatment of infected lesions. We treated this patient according to the first-line treatment plan for HIV patients with Talaromycosis, but considering the low body mass index (BMI) of the patient $\left(18.55 \mathrm{~kg} / \mathrm{m}^{2}\right)$ and drug side effects, we did not utilize a sufficient dose of liposomal amphotericin B. However, as can be seen from the treatment results, the anti-infective therapy was effective, and our patient's symptoms improved significantly.

Ositinib is an optimal medication for treating advanced EGFR mutation-positive lung adenocarcinoma patients with brain metastasis. However, in this case, icotinib was prescribed to the patient due to medical insurance policy and economic considerations. The use of targeted drugs prevents the potential spread of infection caused by chemotherapy. Moreover, simultaneous targeted therapy and anti-infective therapy were found to be safe in this case, which may have been one of the factors leading to the successful treatment of the patient, coupled with the timely diagnosis and treatment of the T. marneffei infection.

In summary, T. marneffei infection is not limited to HIVpositive patients, especially those who reside in endemic regions. Microbiological examination of sputum, BALF, and pleural fluid play an important role in the diagnosis of patients with malignancies, despite few clinical manifestations of infection. Furthermore, missed diagnosis or misdiagnosis of the infection might result in the dissemination of pathogenic microorganisms, especially for those patients who require chemotherapy, radiotherapy, or immunotherapy.

\section{Acknowledgments}

Funding: This work was supported by grants from the Foundation of Priority Project of Science and Technology Plan of Fujian Province (No. 2014Y0037), and the Clinical Key Specialty Construction Project of Fujian Province (No. 2015-593 and No. 2017YZ0001-2).

\section{Footnote}

Reporting Checklist: We present the following article in accordance with the CARE reporting checklist. Available at http://dx.doi.org/10.21037/apm-20-2137

Conflicts of Interest: All authors have completed the ICMJE uniform disclosure form (available at http://dx.doi. org/10.21037/apm-20-2137). The authors have no conflicts of interest to declare.
Ethical Statement: The authors are accountable for all aspects of the work in ensuring that questions related to the accuracy or integrity of any part of the work are appropriately investigated and resolved. All procedures performed in studies involving human participants were in accordance with the ethical standards of the institutional and/or national research committee(s) and with the Helsinki Declaration (as revised in 2013). Written informed consent was obtained from the patient.

Open Access Statement: This is an Open Access article distributed in accordance with the Creative Commons Attribution-NonCommercial-NoDerivs 4.0 International License (CC BY-NC-ND 4.0), which permits the noncommercial replication and distribution of the article with the strict proviso that no changes or edits are made and the original work is properly cited (including links to both the formal publication through the relevant DOI and the license). See: https://creativecommons.org/licenses/by-nc-nd/4.0/.

\section{References}

1. Tsang CC, Lau SKP, Woo PCY. Sixty Years from Segretain's Description: What Have We Learned and Should Learn About the Basic Mycology of Talaromyces marneffei? Mycopathologia 2019;184:721-9.

2. Cao C, Xi L, Chaturvedi V. Talaromycosis (Penicilliosis) Due to Talaromyces (Penicillium) marneffei: Insights into the Clinical Trends of a Major Fungal Disease 60 Years After the Discovery of the Pathogen. Mycopathologia 2019;184:709-20.

3. Chan JF, Lau SK, Yuen KY, et al. Talaromyces (Penicillium) marneffei infection in non-HIV-infected patients. Emerg Microbes Infect 2016;5:e19.

4. Guo J, Li BK, Li TM, et al. Characteristics and Prognosis of Talaromyces marneffei Infection in Non-HIVInfected Children in Southern China. Mycopathologia 2019;184:735-45.

5. Lee PP, Mao H, Yang W, et al. Penicillium marneffei infection and impaired IFN-gamma immunity in humans with autosomal-dominant gain-of-phosphorylation STAT1 mutations. J Allergy Clin Immunol 2014;133:894-6.e5.

6. Atalay A, Koc AN, Akyol G, et al. Pulmonary infection caused by Talaromyces purpurogenus in a patient with multiple myeloma. Infez Med 2016;24:153-7.

7. Zhang J, Huang X, Zhang X, et al. Coinfection of disseminated Talaromyces marneffei and Mycobacteria kansasii in a patient with papillary thyroid cancer: A case 
report. Medicine (Baltimore) 2017;96:e9072.

8. Lin F, Qiu Y, Zeng W, et al. Talaromyces marneffei infection in a lung cancer patient: a rare case report. BMC Infect Dis 2019;19:336.

9. De Monte A, Risso K, Normand AC, et al. Chronic pulmonary penicilliosis due to Penicillium marneffei: late presentation in a french traveler. J Travel Med 2014;21:292-4.

10. Shuangshuang H, Haiyan L, Junru Y, et al. Penicilliosis marneffei of the lung in immunocompetent patients: three cases report and literature review. Chinese Journal of

Cite this article as: Liu W, Xu J, Lin B, Zhang Y, Xie F, Zhou C, Lai G, Zhang L, Yu Z. Pneumonia caused by Talaromyces marneffei in an epidermal growth factor receptor (EGFR) mutation-positive advanced lung adenocarcinoma patient: a case report. Ann Palliat Med 2021;10(1):759-766. doi: 10.21037/apm20-2137
Clinical Infectious Diseases 2018;11:51-5,60.

11. Peng Y, Ping L, Qiu-wen L, et al. Talaromyces marneffei infection complications in an HIV-negative patient with lung cancer: a case report and review of literature. Electronic Journal of Emerging Infectious Diseases 2019;4:169-72.

12. Supparatpinyo K, Khamwan C, Baosoung V, et al. Disseminated Penicillium marneffei infection in southeast Asia. Lancet 1994;344:110-3. 